\title{
Coercion and Compositionality
}

\author{
Giosuè Baggio $^{1 *}$, Travis Choma ${ }^{2 *}$, Michiel van Lambalgen ${ }^{3}$, \\ and Peter Hagoort ${ }^{1,2}$
}

\begin{abstract}
Research in psycholinguistics and in the cognitive neuroscience of language has suggested that semantic and syntactic processing are associated with different neurophysiologic correlates, such as the N400 and the P600 in the ERPs. However, only a handful of studies have investigated the neural basis of the syntax-semantics interface, and even fewer experiments have dealt with the cases in which semantic composition can proceed independently of the syntax. Here we looked into one such casecomplement coercion-using ERPs. We compared sentences such as, "The journalist wrote the article" with "The journalist began the article." The second sentence seems to involve a silent semantic element, which is expressed in the first sentence by
\end{abstract}

\section{INTRODUCTION}

Productivity is the hallmark of language. Speakers of a language are capable of producing and understanding an unlimited number of novel sentences given a finite knowledge base of lexical meanings and syntactic constraints. Productivity requires that the brain be endowed with some computational mechanism combining stored signals into larger, possibly novel expressions. The principle of compositionality has often been invoked to account for the productivity of language (Katz \& Fodor, 1963). Compositionality is the notion that the meaning of a complex expression is a function only of the meanings of its constituent parts and of the way they are syntactically combined (Partee, ter Meulen, \& Wall, 1990). At the processing level, compositionality seems to imply that, if word meanings are assembled based on syntactic constraints alone, then there is no other source of combinatoriality in language than the syntax. However, recent psycholinguistic work suggest that language comprehension cannot be reduced to a strict, syntax-driven composition of lexical meanings, but involves an independent level of semantic combinatorics which can, in some cases, constrain syntactic analysis (Kim \& Osterhout, 2005), and uses world knowledge and discourse context-beyond context-invariant word meanings - to arrive at a full inter-

\footnotetext{
${ }^{1}$ Max Planck Institute for Psycholinguistics, Nijmegen, The Netherlands, ${ }^{2}$ Radboud University, Nijmegen, The Netherlands, ${ }^{3}$ University of Amsterdam, The Netherlands

* Listed in alphabetical order. The first two authors contributed equally to the present study.
}

the head of the verb phrase (VP) "wrote the article." The second type of construction may therefore require the reader to infer or recover from memory a richer event sense of the VP "began the article," such as began writing the article, and to integrate that into a semantic representation of the sentence. This operation is referred to as "complement coercion." Consistently with earlier reading time, eye tracking, and MEG studies, we found traces of such additional computations in the ERPs: Coercion gives rise to a long-lasting negative shift, which differs at least in duration from a standard N400 effect. Issues regarding the nature of the computation involved are discussed in the light of a neurocognitive model of language processing and a formal semantic analysis of coercion. pretation of the utterance (Hagoort \& van Berkum, 2007). In this article, ERP data will be presented that, taken together with earlier psycholinguistic findings and theoretical considerations (Baggio, van Lambalgen, \& Hagoort, in press; Pylkkänen, 2008), seem to challenge a strongly compositional account of meaning assembly.

\section{Complement Coercion and Enriched Semantic Composition}

A great deal of the experimental evidence supporting the independence of semantic operations has come from studies of complement coercion (Pylkkänen \& McElree, 2007; McElree, Pylkkanen, Pickering, \& Traxler, 2006; Traxler, McElree, Williams, \& Pickering, 2005; Traxler, Pickering, \& McElree, 2002; McElree, Traxler, Pickering, Seely, \& Jackendoff, 2001). Verbs such as "begin" can occur in two types of syntactic constructions with similar meanings: as a subject control verb with a noun phrase (NP) and a verb phrase (VP) as arguments, like in "The journalist began to write the article" or "The journalist began writing the article"; or as a transitive verb with two NP arguments, like in "The journalist began the article" (Egg, 2003). In a number of cases, constructions of the second type seem to involve an implicit semantic element, which is expressed in the first type by the head of the VP argument. The contrast between silent and expressed meaning can also be found in the following pair:

(1) The journalist wrote the article before his coffee break.

(2) The journalist began the article before his coffee break. 
In (1), the activity that the journalist is performing is expressed by the verb "wrote," whereas in (2) the activity which was initiated by the journalist (presumably, writing) is not expressed by any of the sentence's constituent expressions. Rather, the implicit meaning in (2) is the result of an inference-an operation that is not syntactic in nature - to the effect that, because journalists typically write articles, the sentence means that the journalist began writing (or typing, etc.) the article. The semantic ambiguity of expressions such as "began the article" will be briefly discussed later on. Suffice it to say here that these constructions are hypothesized to involve some form of enriched composition, whereby the meaning that is computed on-line contains more information than can be derived from word meanings and the syntax. The result of enriched composition is a model that includes a representation of the writing activity implicit in "began the article."

\section{Reading Time, Eye Tracking, and MEG Studies of Complement Coercion}

McElree et al. (2001) were the first to report longer selfpaced reading times at the noun ("article") and at the noun + 1 ("before") positions in complement coercions (2) compared to controls (1). McElree et al. (2006) observed that coercing sentences are interpreted less accurately, that is, more errors were made in an on-line sensicality judgment task, and more slowly than control sentences. Traxler et al. (2002) found that coercing constructions result in longer total reading times as well as eye fixations on and regressions from the noun phrase region compared to control sentences. To rule out the possibility that processing differences are due to some property of verbs like "begin," such as, for instance, their syntactic polyvalence as characterized above or semantic ambiguity (Frisson \& McElree, 2008; McElree et al., 2006; Traxler et al., 2005), the authors compared (1) and (2) to sentences like (3), in which a noun denoting an event occurs as a complement:

(3) The journalist began the meeting before his coffee break.

Here, the activity initiated by the journalist is expressed by the noun phrase "the meeting." If processing difficulty is due to the verb "begin," there should be no difference in reading times between (2) and (3). If, however, increased processing costs are due to recovering or inferring silent meaning, (2) should still result in longer reading times compared to (3). Complements denoting entities (i.e., "article") indeed result in longer second pass and total reading times in the critical NP region compared to event-denoting complements (i.e., "meeting"). This suggests that readers experience difficulty only when the appropriate event sense is not encoded in the lexical items, but must be inferred.
Traxler et al. (2005) propose that coercion involves four processing steps that unfold sequentially after the reader encounters the complement. Following type-shifting accounts of coercion (Pustejovsky, 1995), their claim is that the complement "the article" violates the verb's semantic requirements, as "begin" should be followed by an expression denoting an event such as "the meeting" or "writing the article." In this framework, enriched composition consists in shifting the semantic type of the complement NP such that an entity-denoting expression is used to refer to an event instead (see Discussion section for an alternative account to type-shifting). Here are the steps involved in the coercion process:

1. The noun's lexical entry is accessed and an attempt is made to integrate the stored senses into the semantic representation on-line;

2. The mismatch between the verb's semantic requirements and the stored lexical senses of the noun triggers a coercion computation (Steps 3 and 4);

3. Properties associated with the noun and properties associated with the discourse are used to infer the correct event sense related to the entity-denoting complement;

4. The event sense is incorporated into the semantic representation of the discourse by reconfiguring the semantic representation of the complement from that of the entity to that of the related event.

The authors argue that the processing difficulties observed in the experiments of McElree et al. (2001, 2006) and Traxler et al. (2002) result from Step 4 of the proposed model. Traxler et al. (2005) present convincing evidence for this using different sentence types and contextual manipulations.

Further evidence that coercing constructions incur in definite processing costs was recently collected using MEG. Pylkkänen and McElree (2007) recorded event-related fields (ERFs) while participants read sentences like (1), (2), and (6) word-by-word.

(6) The journalist astonished the article before his coffee break.

Multiple-source models were used to account for ERFs elicited by critical nouns. Semantically anomalous nouns, such as "article" in (6), modulate activity in left temporal cortices, resulting in stronger source amplitudes compared to coercing and control sentences, peaking around 350 msec following noun onset. By contrast, coercing nouns, such as "article" in (2), elicit stronger activation at anterior midline sources. For this reason, the effect of coercion is referred to by Pylkkänen and McElree (2007) as the anterior midline field (AMF). This study suggests that there is a dissociation in the brain areas involved in different stages of meaning assembly: Lexico-semantic processes, indexed by the N400 in the ERPs (Kutas \& Hillyard, 1980) and the M350 in the ERFs, seem to be carried out largely in left 
temporal cortices (Halgren et al., 2002; Helenius, Salmelin, Service, \& Connolly, 1998; Simos, Basile, \& Papanicolaou, 1997), whereas aspects of meaning composition beyond the word level may involve medial frontal cortices (Pylkkänen \& McElree, 2007).

\section{The Present ERP Study}

The experiments just reviewed indicate that coercing expressions result in definite processing costs. However, connecting those findings to current knowledge of the processing mechanisms (as opposed to the functional anatomy) of language in the brain is problematic. Although ERPs are no longer the only in vivo measure of neural responses to linguistic stimuli, a significant portion of that knowledge has come and still comes from ERP studies. There currently is no EEG work on complement coercion, and moreover, linking the MEG findings of Pylkkänen and McElree (2007) to the ERP literature on semantic processing is quite possible, but not straightforward.

It is becoming increasingly clear that the N400 (Kutas \& Hillyard, 1980) is not the whole story as far as the neurophysiologic correlates of semantic integration are concerned. Still, the component remains one measure of semantic processing load, and an important term of comparison when brain correlates of different aspects of semantic composition are sought. This entails that experiments investigating enriched composition would benefit from being able to directly compare the responses evoked by complement coercion with a standard N400 effect. Such a comparison seems possible on the basis of MEG data only indirectly. Coercing and anomalous nouns modulate responses at different MEG sources, and this implies functional differences between processing semantic anomalies and coercing constructions. However, AMF modulations were discovered only recently (Pylkkänen \& McElree, 2007); they do not seem to surface with equal strength across subjects and experimental paradigms (Brennan \& Pylkkänen, 2008; Pylkkänen \& McElree, 2007), and they are not found in all putative cases of enriched composition, such as concealed questions (Harris, Pylkkänen, McElree, \& Frisson, 2007). Because ERPs afford a direct comparison with the wellestablished N400, they might provide more reliable evidence for, or against, a functional dissociation between processing semantic anomalies and complement coercions. That is the rationale behind the subsequently presented ERP study.

\section{METHODS}

\section{Participants}

Twenty-one right-handed native English speakers (9 women, age range $=18-34$ years) participated in the experiment. Dialects spoken by participants included American, Canadian, Australian, and British varieties of English. All participants were nationals of English-speaking countries (USA, UK, Canada, Australia), spending a short period for work or study purposes in the Netherlands. The same applies to the subjects recruited for the norming studies (see below). All participants had normal or corrected-to-normal vision and no history of neurological, psychiatric, or cognitive disorders. Subjects gave their informed consent prior to each session and were paid for taking part in this study. Three subjects ( 1 woman) were left out of the final analysis due to a high number $(>20 \%)$ of trials contaminated by artifacts.

\section{Materials}

The set of materials consisted of 159 triplets like the following:

Coercing: The journalist began the article before his coffee break.

Anomalous: The journalist astonished the article before his coffee break.

Neutral: The journalist wrote the article before his coffee break.

The coercing verbs used and their frequencies within the stimulus set were: "begin" (15), "finish" (27), "start" (25), "try" (19), "attempt" (3), "master" (12), "endure" (6), "complete" (20), "enjoy" (28), "manage" (1), and "resist" (3) (see Traxler et al., 2002, 2005; McElree et al., 2001 for similar choices). From the original set of 159 triplets, three test versions were constructed, each containing 53 items per condition.

Each version included also 150 fillers, which were identical across versions. Fillers were sentences of varying structure, content, and length, and included coercing and anomalous sentences with the critical region in different positions in the sentence structure. Coercing and anomalous fillers were added so that readers could not anticipate where in the sentence they would encounter the coercing or anomalous noun. Each participant read 159 critical sentences (53 per condition) and 150 fillers.

The default event sense associated with each coercing expression was determined using a fill-in-the-blank test administered to 25 subjects, with 160 items such as: "The journalist began the article before his coffee break." Conflating synonyms and near-synonyms, on average, 75\% of the respondents selected the same event sense, which was subsequently used as the verb in the corresponding neutral items. A second pretest was aimed at matching the mean frequencies of verbs across conditions. Using the CELEX corpus for English (Baayen, Piepenbrock, \& Gulikers, 1996), lemma frequencies per million words were: coercing, 314.7; anomalous, 255; neutral, 299.8 (all comparisons using $t$ tests, $p>.05$ ). Word-form frequencies per million words were: coercing, 31.1; anomalous, 26.6; neutral, 23.6 (all comparisons using $t$ tests, $p>.05$ ). Verb length was matched across conditions: coercing, 6.74; anomalous, 6.52; neutral, 6.44 (all comparisons using $t$ tests, $p>.05)$. A third pretest was carried out to match the cloze 
probabilities of the object nouns in neutral and coercing sentences. The cloze probability of a particular word is defined as the frequency with which it occurs in the overall response set at a particular position in a sentence. A fill-in-the-blank test was administered to 40 participants, using 159 sentences per condition: "The journalist began the __ or "The journalist wrote the __. Nouns were selected for use in the ERP stimuli that were equiprobable in the neutral and coercing cases based on the survey results. The mean cloze probabilities after adjustment were .061 for the coercing and .086 for the neutral condition ( $t$ test, $p>.05$ ). Cloze probabilities were 0 for the anomalous case: Nouns (such as "article" following "The journalist astonished the") were strongly dispreferred and, therefore, likely to be processed as anomalous.

\section{Procedure}

After applying the electrodes (see Recording section), participants were conducted into the experimental booth and were asked to sit in front of the video monitor. Subjects were given written instructions to avoid eye blinks and movements during the presentation of the stimuli. They were also directed to read the sentences carefully, and they were informed that the experimenter would ask them some questions at the end of the session. Stimulus sentences were presented one word at a time for 300 msec per word, followed by 300 msec of blank screen before the onset of the next word. Words were presented in white on a black background in the center of the screen. After 1000 msec of blank screen following the offset of the last word of each sentence, an asterisk mark appeared for $1500 \mathrm{msec}$, during which subjects were allowed to blink.

\section{Recording}

EEG and EOG signals were recorded using $\mathrm{Ag} / \mathrm{AgCl}$ electrodes. The EOG was measured from four electrodes: one at the outer canthus of each eye, one below and one above (Fp1) the left eye. The EEG was measured from 28 scalp electrodes, which were arranged according to American Electrophysiological Society conventions: Fp1, Fp2, F7, F3, Fz, F4, F8, FC5, FC1, FCz, FC2, FC6, T7, C3, Cz, C4, T8, CP5, CP1, CP2, CP6, P7, P3, Pz, P4, P8, O1, O2. Two additional electrodes were placed on the left and right mastoids, the former serving as the reference during the measurement. All EEG and EOG electrodes were rereferenced off-line to a linked mastoid. EEG electrodes were attached to an elastic cap, whereas EOG and reference electrodes were applied using two-sided adhesive decals external to the cap. Electrode impedance was kept below $5 \mathrm{k} \Omega$ throughout the experiment. The EEG/EOG was amplified using a multichannel BrainAmp DC system, with a 500-Hz sampling rate, a low-pass filter set at $100 \mathrm{~Hz}$, and a 10-sec time constant.

\section{Data Analysis}

Data analysis was conducted using FieldTrip, ${ }^{1}$ a MATLAB package for processing EEG signals. The following transforms were applied to each subject's dataset. Segments corresponding to the determiner and the noun were extracted from the EEG with an interval of 200 msec preceding and $1000 \mathrm{msec}$ following stimulus onset. Baseline correction used the 200-msec interval preceding the onset of the stimulus. Artifact rejection was based on two FieldTrip functions: The first detects and rejects all trials that contain activity exceeding a threshold of $\pm 100 \mu \mathrm{V}$; the second identifies and discards trials contaminated with eye movements or blinks by means of thresholding the $z$-transformed value of the raw data in the EOG channels, preprocessed using a band-pass filter of 1-15 Hz.

To test for significant differences between conditions in the time windows of interest, we used repeated measures ANOVA with two within-subject factors: experimental condition (coercing/control/anomalous) and electrode site (28 levels, one for each scalp electrode). For the contrasts between condition pairs, a similar analysis was carried out, in which the factor experimental condition had two levels only. ANOVAs were carried out in two time windows: 300-500 msec, corresponding to the latency of the N400; and 700-1000 msec, based upon a visual inspection of ERP waveforms (see Results section below).

To characterize the temporal profiles of the ERP effects elicited by coercing, anomalous, and neutral nouns, we used a nonparametric randomization procedure for $t$ statistics (Maris \& Oostenveld, 2007; Maris, 2004). The algorithm uses mean amplitude $(\mu \mathrm{V})$ values in each condition for time bins of $100 \mathrm{msec}$, starting from the onset of the word and ending $1000 \mathrm{msec}$ after. The output is a cluster of electrodes in which differences between two conditions are significant in each time bin, the sum of $t$ statistics in that cluster, and Monte Carlo estimates of $p$ values.

\section{RESULTS}

An inspection of ERP waveforms elicited by the determiner (data not shown) and the noun (Figure 1) revealed no differences between conditions in the early ERP components such as the N1 and P2 (in both cases: 0-150 msec, $p>.1$; 150-300 msec, $p>$.1). The ERP modulations evoked by the determiner do not differ across conditions in later time windows either. This conclusion is supported by the results of ANOVA statistics in the standard N400 and P600 time windows (data not shown; 300-550 msec, $p>.1$; 550-900 msec, $p>$.1).

Differences emerge only around $300 \mathrm{msec}$ following the onset of the critical noun (Figure 1). The noun in the anomalous condition elicits a larger N400 compared to the same lexical item in the control condition. Similarly, the coercing noun evokes what appears to be a larger N400 compared to the control noun. Both effects start around $300 \mathrm{msec}$ following word onset, peak approximately $100 \mathrm{msec}$ 
Figure 1. (A) Grand-average $(n=18)$ topographies displaying the mean amplitude differences between the ERPs evoked by coercing versus neutral nouns and anomalous versus neutral nouns, in the $300-550 \mathrm{msec}$ and $700-1000$ msec regions following noun onset.

(B) Grand-average $(n=18)$ waveforms from frontal, central, and parietal electrode sites time-locked to the onset (0 msec) of the noun. Negative values are plotted upward.

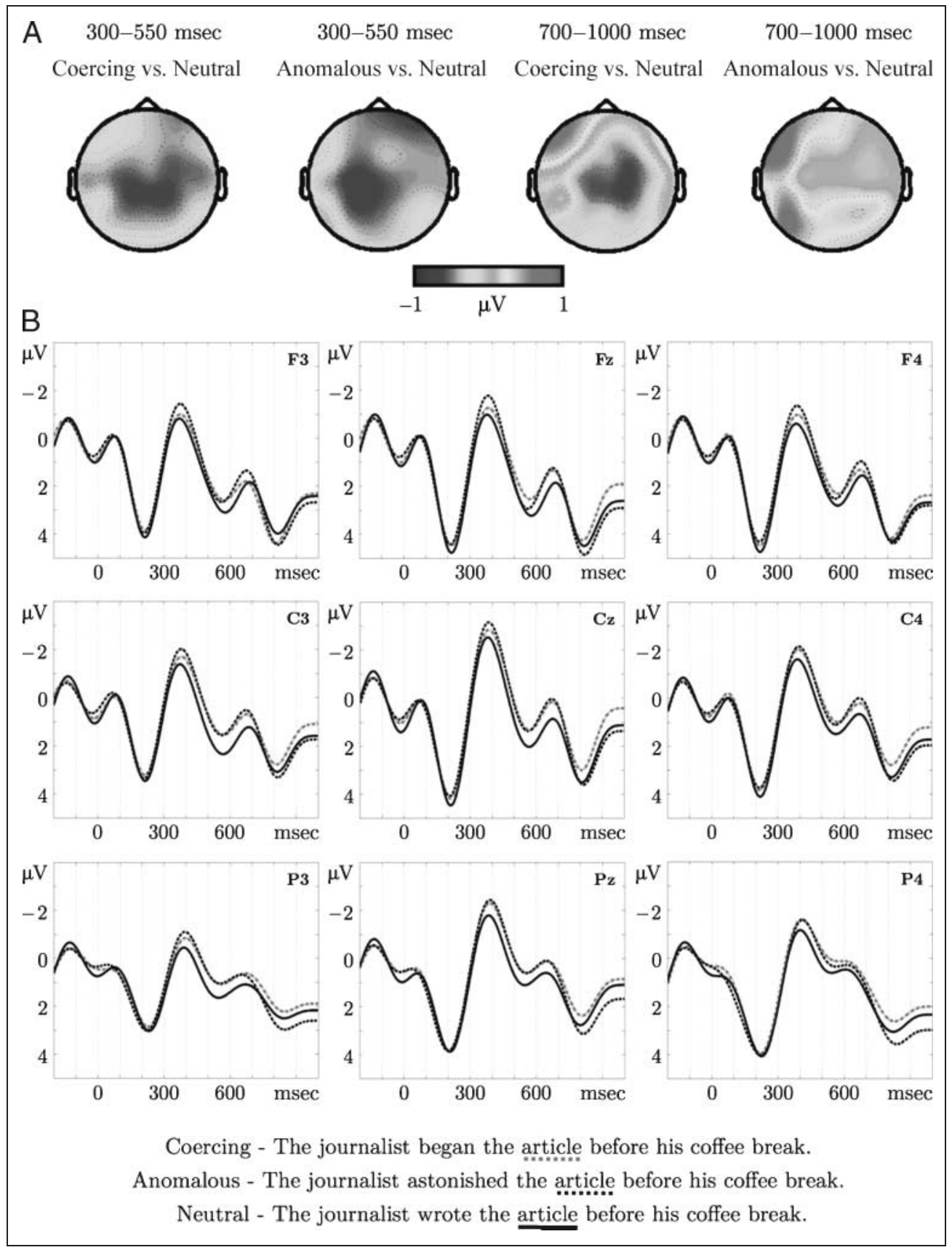

later, and have a centro-parietal distribution that is characteristic of the N400. In both cases, the N400 is statistically reliable in the 300-550 msec time window (Table 1). In this interval, there is no significant difference between the effects evoked by anomalous and coercing nouns.

An examination of ERP waveforms (Figure 1B) suggests that brain potentials specific to coercion-in which the coercing condition differs from the neutral and the anomalous cases-surface in a later time window, starting at about $700 \mathrm{msec}$ from noun onset. Coercing nouns evoke a negative-going shift with a central scalp distribution (Figure 1), lasting until the end of the epoch (1000 msec). The topography of this negativity resembles that of the $\mathrm{N} 400$ effect evoked by coercing nouns in the $300-550 \mathrm{msec}$ in- terval (Figure 1A; compare the first and third plots). This later effect, too, is statistically highly significant compared to the control condition (Table 1), and approaches significance compared to the anomalous case $(p<.1$; see below for a more sensitive test using cluster-and-randomization analysis). The difference between anomalous and control nouns is, in this time interval (700-1000 msec), not significant.

Due to the resemblance in scalp topography between the earlier and the later negativities evoked by coercion (Figure $1 \mathrm{~A}$ ), and the seemingly continuous negative shift in the waveforms (Figure 1B), it may be suggested that the early negative shift is sustained until the end of the epoch. Cluster-and-randomization analyses indicate that this is most likely the case. Whereas anomalous nouns, compared to 
Table 1. Summary of ANOVA Statistics for the ERP Analysis

\begin{tabular}{lrc}
\hline & $300-550$ msec & $700-1000$ msec \\
\hline Condition & $F(1,17)=3.948^{*}$ & $F(1,17)=13.600^{* * * *}$ \\
$\begin{array}{l}\text { Coercing vs. } \\
\quad \text { Neutral }\end{array}$ & $F(1,17)=4.6245^{*}$ & $F(1,17)=14.925 * * *$ \\
$\begin{array}{l}\text { Anomalous vs. } \\
\quad \text { Neutral }\end{array}$ & $F(1,17)=4.5411^{*}$ & $F(1,17)=2.8552$ \\
$\begin{array}{l}\text { Coercing vs. } \\
\text { Anomalous }\end{array}$ & $F(1,17)=0.0441$ & $F(1,17)=3.7095$ \\
\hline$* p<.05$. & \\
$* * * p<.001$.
\end{tabular}

neutral nouns, give rise to statistically significant activity in a centro-parietal cluster of electrodes (sites: C3, Cz, CP5, CP1, P3, Pz) limited to the 300-500 msec interval (see Table 2), coercing nouns modulate activity in similar scalp regions (electrodes: C3, Cz, C4, CP1, CP2, P3, P4), starting at $300 \mathrm{msec}$ and lasting until the end of the epoch (Table 2). The difference between anomalous and coercing nouns is significant after $700 \mathrm{msec}$ (Table 2; compare the coercion vs. anomalous contrasts in Tables 1 and 2; see Maris \& Oostenveld, 2007 and Maris, 2004 for a discussion of sensitivity issues).

\section{DISCUSSION}

Our results provide further support to the claim that complement coercion gives rise to definite processing costs - a claim that is now backed by reading time, eye tracking, MEG, and EEG data. It can be concluded that, with respect to neutral items, coercing sentences seem to require additional computations (whatever their exact nature), as is shown by reliable differences in the coercion vs. neutral contrasts. Moreover, processing the coercing noun leads to downstream integration costs, as is indicated by the coercion versus anomalous contrast in later time windows. The ERP effects of coercion differ from those of semantic anomaly at least in their temporal profile. However, it cannot be concluded that the sustained negativity evoked by coercion and the N400 elicited by anomaly are entirely different effects, as the former could also be seen as a sequence of N400s evoked by the coercing noun and by the word following the noun.

Regardless of the exact physiological characterization of the effect elicited by coercion and its functional interpretation, it seems that the observed negativity should be attributed to some stage of the coercion operation. For instance, the effect can hardly be explained by lexical repetition of coercing verbs across stimulus sentences. As was shown by Rugg (1983), ERPs to repeated words evoke an early (around $200 \mathrm{msec}$ ) transient negativity and a later, topographically widespread and temporally sustained positive shift. Bresson, Kutas, and van Petten (1992) showed that repetition reduced the amplitude and shortened the duration of the N400. In our study, the exact opposite pattern of effects was found. Moreover, our norming studies (see Materials section) seem to provide some assurance that alternative explanations of the ERP effects of coercion in terms of event sense ambiguity, word frequency, and cloze probability can be ruled out.

\section{Enriched Composition vs. Simple Composition}

In this article, we have presented ERP data that, in line with earlier reading time, eye tracking, and MEG studies, suggest that some form of enriched semantic composition may be involved in processing sentences in which verbs such as "begin" are used transitively with two NP arguments, like in "The journalist began the article." The neural reality of complement coercion, which has now found some support with both MEG (Pylkkänen \& McElree, 2007) and EEG, seems to challenge linguistic theories that analyze these constructions in a strongly

Table 2. Summary of Cluster-and-Randomization Statistics for the ERP Analysis

\begin{tabular}{|c|c|c|c|}
\hline & Coercing vs. Neutral & Anomalous vs. Neutral & Coercing vs. Anomalous \\
\hline 300-400 msec & $t(16)=-54.874 * *$ & $t(16)=-37.412 *$ & \\
\hline $400-500 \mathrm{msec}$ & $t(16)=-61.287^{* *}$ & $t(16)=-34.018 *$ & \\
\hline 500-600 msec & $t(16)=-52.658 * *$ & & \\
\hline 600-700 msec & $t(16)=-50.151 *$ & & \\
\hline 700-800 msec & $t(16)=-44.456^{*}$ & & $t(16)=-20.135^{*}$ \\
\hline 800-900 msec & $t(16)=-53.645^{* *}$ & & $t(16)=-35.419 * *$ \\
\hline 900-1000 msec & $t(16)=-64.083^{* *}$ & & $t(16)=-63.260 * *$ \\
\hline
\end{tabular}

The first significant effects occurred at 300-400 msec. Empty cells denote the absence of significant clusters.

$* p<.05$.

$* * p<.01$. 
compositional way. Some of these accounts would attribute the complexity of coercing expressions to interpreting an unpronounced syntactic head (i.e., a silent V node in the syntactic structure of a coercing sentence), rather than to inferring a missing semantic element. It is quite possible that earlier behavioral and MEG findings, and the present ERP results, can be accommodated in that sort of framework. However, at least as far as ERP data are concerned, it would seem that the syntactic account would be more consistent with a different response than the negativity reported above, possibly a modulation of the P600, which has been associated also with syntactic complexity, and not just with syntactic violations (Hagoort, 2003). Reasons in favor of a semantic account of coercion are theoretical parsimony (for the syntactic story has to postulate operations at both syntactic and semantic levels) and linguistic considerations (for a discussion of these issues, see Baggio et al., to appear; Pylkkänen, 2008; Pylkkänen \& McElree, 2006; for some criticism of theories postulating unpronounced syntactic elements, see Culicover \& Jackendoff, 2005).

Theories exist that model the coercion process in a noncompositional manner, such that world knowledge is recruited alongside lexical semantics and the syntax. One such proposal is based on unification, a mechanism adopted by a number of linguistic accounts of syntactic and semantic structure assembly (van Lambalgen \& Hamm, 2004; Vosse \& Kempen, 2000; Joshi \& Schabes, 1997). Although equally viable approaches have been developed that do not rely on unification (Jackendoff, 1997; Pustejovsky, 1995), what makes that concept appealing here is that models of language processing inspired by neurobiology are trying to bridge the gap between linguistics and neuroscience precisely by means of the notion of unification (Hagoort, Baggio, \& Willems, 2009; Hagoort, 2003, 2005). Moreover, complement coercion can be described in a unification-based theory of tense, aspect, and event structure, such as the Event Calculus (van Lambalgen \& Hamm, 2004). This formalism is alternative to analyses based on types and type-shifting (Pylkkänen \& McElree, 2006; Pustejovsky, 1995), in which earlier experimental research was grounded (Pylkkänen \& McElree, 2007; McElree et al., 2001, 2006; Traxler et al., 2002, 2005).

In the Event Calculus, the verb "begin" and its synonyms are represented by a clause of the form Initiates (start, $a, t$ ), which means that a starting event (start) initiates an activity $a$ at time $t$. The variable $a$ has to be unified with material provided by the discourse context or world knowledge, that is, a VP ( $a=$ write) or an NP denoting an event ( $a=$ meeting). What makes this formalism attractive is its cognitive plausibility (van Lambalgen \& Hamm, 2004). That is, semantic representations for verbs such as Initiates(start, $a, t$ ) and the unification operation itself find their motivation in the evolutionary link between the semantics of tense, aspect, and event structure on the one hand, and planning on the other (Steedman, 2002). The Event Calculus is, in fact, a plan- ning formalism, which has found successful applications to the semantics of tense, aspect, and event structure (Hamm, Kamp, \& van Lambalgen, 2006; van Lambalgen \& Hamm, 2004). In brief, three arguments justify our preference for unification over type-shifting as a framework in which to try to make sense of experimental data: A link with the notions of unification and binding in neuroscience, cognitive plausibility through a connection to planning and an ontology that does not involve a hierarchy of types, and success in modeling linguistic phenomena.

It should be emphasized that the ERP data reported here do not favor unification over alternative accounts of coercion such as Pustejovsky's (1995) type-shifting analysis, nor was the present ERP study designed to test predictions that are licensed by the unification model. However, and conversely, the unification framework can be used to shed some light on our ERP data. The Event Calculus, in particular, suggests that coercing sentences require more complex unification operations than neutral sentences, as an extra inference step has to be made in order to unify the variable $a$ with a suitable activity. The goal of this inference step is precisely to find out an activity that suits semantically the given discourse context. As can be seen, unification provides a tentative formalization of the traditional notion of the "integration" of lexical items into a sentence or a discourse model. Therefore, to the extent that the N400 can be taken as an index of semantic integration (see below), the N400-like shift elicited by the coercing noun may be associated with more complex, inference-driven unifications leading to a richer event sense. The later negativity, or sequence of N400 effects, could reflect difficulty in integrating lexical items following the noun into a complex semantic representation of the unfolding sentence. The type-shifting account formalizes the traditional notion of integration in different terms, but in this case, it may not lead to an entirely different account of the data. It is an interesting question whether linguistic phenomena exist for which unification and typeshifting make different processing predictions, and whether such predictions can be tested experimentally.

\section{Enriched Composition vs. Lexico-semantic Anomaly}

Pylkkänen and McElree (2007) correctly point out that the nature of the N400 as an index of integration difficulty has so far not been demonstrated. More precisely, experimental evidence points in the direction of two competing accounts: The first, which postulates that the N400 is directly correlated with the difficulty in integrating the meaning of the upcoming word in the sentence context (Li, Hagoort, \& Yang, 2008; Ferretti, Kutas, \& McRae, 2007; Hald, SteenbeekPlanting, \& Hagoort, 2007; Özyürek, Willems, Kita, \& Hagoort, 2007; Nieuwland \& van Berkum, 2005; Hagoort, Hald, Bastiaansen, \& Petersson, 2004; van Berkum, Brown, Hagoort, \& Zwitserlood, 2003; van Berkum, Zwitserlood, 
Hagoort, \& Brown, 2003; van Berkum, Brown, \& Hagoort, 1999; van Berkum, Hagoort, \& Brown, 1999); the second, which proposes the N400 is inversely correlated with the amount of preactivation a word receives from the preceding context (De Long, Urbach, \& Kutas, 2005; Kutas \& Federmeier, 2000; Federmeier \& Kutas, 1999; Hagoort \& Brown, 1994; Holcomb, 1993; van Petten \& Kutas, 1990, 1991; Kutas \& Hillyard, 1980, 1984). It is important to avoid setting up a false opposition because these two accounts merely highlight different processes that may underlie the generation of the N400, but are, in no way, alternative and mutually exclusive theories. In fact, a unified view that fits most data seems conceivable, where different roles are assigned to each cerebral hemisphere: Predictive semantic coding may be seen as a left hemisphere mechanism, whereas the right hemisphere contribution could be strictly postlexical in nature, contributing to the integration of word meanings, that is, to unification (Hagoort et al., 2009; Kutas \& Federmeier, 2000; Federmeier \& Kutas, 1999).

It seems possible to argue that, even if such a unified view of the N400 was correct, this would not destroy our preferred interpretation of the ERP correlates of coercion. According to the preactivation view, the amplitude of the N400 component is inversely correlated with the amount of lexico-semantic priming a word receives from the preceding sentence or discourse context (Kutas \& Federmeier, 2000; Federmeier \& Kutas, 1999). One driving factor behind preactivation is semantic relatedness, thus "article" should be preactivated more strongly by the fragment "The journalist wrote the" as compared to both "The journalist astonished the" and "The journalist began the." However, the cloze-probability data reported in Materials section show that there is little difference between neutral ("the journalist wrote the") and coercing ("the journalist began the") contexts as far as the expectancy of the noun "article" is concerned. This seems to challenge an account of the ERP correlates of coercion that is based on lexical preactivation alone. As a result, it lends some plausibility to the unification view expounded above.

Complement coercion involves inferring a plausible activity based on world knowledge, and integrating it (via unification or type-shifting) into the event structure of the VP "began the article." This process, which seems to be noncompositional in nature, has two brain correlates: modulations at sources different from those that generate the N400, as the AMF data by Pylkkänen and McElree (2007) show; and modulations of the N400 at the coercing noun and more downstream regions. Our ERP data do not license the conclusion that the neural processes underlying enriched composition are physiologically or functionally distinct from those that support standard semantic composition and the processing of semantic anomalies. We did find a specific ERP effect of coercion, but this differed from a standard N400 only in duration: a sustained negative shift or a sequence of
N400s to subsequent words was found, with a scalp distribution that is similar to that of a standard N400. Contrary to what one might have expected on the basis of the MEG study, the ERP data do not distinguish between anomaly and coercion in the typical N400 window, or in the time window in which the AMF was found (350$500 \mathrm{msec}$ ). Based on the data available so far, it is impossible to say how exactly, if at all, the AMF, the N400, and the later negativity evoked by coercion are related. Further MEG, EEG, and, possibly, fMRI studies into enriched composition are necessary to address this issue. Additional experimental testing might also be useful to address the intriguing suggestion, made by Pylkkänen and McElree (2007), that the physiologic responses specific to coercion - the AMF in their report — may be generated in medial prefrontal cortices, which have been associated with "perspective taking" and inferring others' mental states. The interpretation of coercing sentences, and the inference to find out a plausible activity, might involve taking the perspective of the agent depicted in the sentence (i.e., the journalist). EEG data might reveal whether the N400 is sensitive to varying degrees of complexity in perspective taking during sentence processing, and fMRI studies might result in a more precise picture of the brain structures recruited by noncompositional meaning assembly.

\section{Acknowledgments}

We thank Oliver Bott, Fritz Hamm, Janina Radö, Jos van Berkum, and three anonymous reviewers for useful comments and suggestions on earlier versions of the manuscript. We also thank the Netherlands Organization for Scientific Research (NWO) for support under grant 051.04.040.

Reprint requests should be sent to Giosuè Baggio, Max Planck Institute for Psycholinguistics, P.O. Box 310, 6500 AH Nijmegen, The Netherlands, or via e-mail: giosue.baggio@mpi.nl.

\section{Note}

1. For more information, see http://fieldtrip.fcdonders.nl/.

\section{REFERENCES}

Baayen, R. H., Piepenbrock, R., \& Gulikers, L. (1996). CELEX2. Philadelphia: Linguistic Data Consortium.

Baggio, G., van Lambalgen, M., \& Hagoort, P. (in press). The processing consequences of compositionality. In W. Hinzen, E. Machery, \& M. Werning (Eds.), The Oxford handbook of compositionality. Oxford University Press.

Brennan, J., \& Pylkkänen, L. (2008). Processing events: Behavioral and neuromagnetic correlates of aspectual coercion. Brain and Language, 106, 132-143.

Bresson, M., Kutas, M., \& van Petten, C. (1992). An event-related potential (ERP) analysis of semantic congruity and repetition effects in sentences. Journal of Cognitive Neuroscience, 4, 132-149.

Culicover, P. W., \& Jackendoff, R. (2005). Simpler syntax. Oxford: Oxford University Press. 
De Long, K. A., Urbach, T. P., \& Kutas, M. (2005). Probabilistic word pre-activation during language comprehension inferred from electrical brain activity. Nature Neuroscience, 8, 1117-1121.

Egg, M. (2003). Beginning novels and finishing hamburgers: Remarks on the semantics of "to begin." Journal of Semantics, 20, 163-191.

Federmeier, K. D., \& Kutas, M. (1999). Right words and left words: Electrophysiological evidence for hemispheric differences in meaning processing. Cognitive Brain Research, 8, 373-392.

Ferretti, T. R., Kutas, M., \& McRae, K. (2007). Verb aspect and the activation of event knowledge. Journal of Experimental Psychology: Learning, Memory, and Cognition, 33, 182-196.

Frisson, S., \& McElree, B. (2008). Complement coercion is not ambiguity resolution: Evidence from eye movements. Journal of Experimental Psychology: Learning, Memory, and Cognition, 31, 1-11.

Hagoort, P. (2003). How the brain solves the binding problem for language: A neurocomputational model of syntactic processing. Neuroimage, 20, S18-S29.

Hagoort, P. (2005). On Broca, brain, and binding: A new framework. Trends in Cognitive Sciences, 9, 416-423.

Hagoort, P., Baggio, G., \& Willems, R. M. (2009). Semantic unification. In M. Gazzaniga (Ed.), The new cognitive neurosciences. Cambridge, MA: MIT Press.

Hagoort, P., \& Brown, C. (1994). Brain responses to lexical ambiguity resolution and parsing. In C. Clifton, L. Frazier, \& K. Rayner (Eds.), Perspectives on sentence processing (pp. 45-81). Hillsdale, NJ: Erlbaum.

Hagoort, P., Hald, L., Bastiaansen, M., \& Petersson, K. M. (2004). Integration of word meaning and world knowledge in language comprehension. Science, 304, 438-441.

Hagoort, P., \& van Berkum, J. (2007). Beyond the sentence given. Philosophical Transactions of the Royal Society of London, Series B, Biological Sciences, 362, 801-811.

Hald, L., Steenbeek-Planting, E., \& Hagoort, P. (2007). The integration of discourse context and world knowledge in online sentence comprehension. Evidence from the N400. Brain Research, 1146, 210-218.

Halgren, E., Dhond, R. P., Christensen, N., Van Petten, C., Marinkovic, K., Lewine, J. D., et al. (2002). N400-like MEG responses modulated by semantic context, word frequency, and lexical class in sentences. Neuroimage, 17, 1101-1116.

Hamm, F., Kamp, H., \& van Lambalgen, M. (2006). There is no opposition between formal and cognitive semantics. Theoretical Linguistics, 22, 1-40.

Harris, J., Pylkkänen, L., McElree, B., \& Frisson, S. (2007). The cost of question concealment: Eye-tracking and MEG evidence. Brain and Language, 107, 44-61.

Helenius, P., Salmelin, R., Service, E., \& Connolly, J. (1998). Distinct time courses of word and context comprehension in the left temporal cortex. Brain, 121, 1133-1142.

Holcomb, P. (1993). Semantic priming and stimulus degradation: Implications for the role of the N400 in language processing. Psychophysiology, 30, 47-61.

Jackendoff, R. (1997). The architecture of the language faculty. Cambridge, MA: MIT Press.

Joshi, A., \& Schabes, Y. (1997). Tree-adjoining grammars. In A. Salomma \& G. Rosenberg (Eds.), Handbook of formal languages and automata. Berlin: Springer-Verlag.

Katz, J. J., \& Fodor, J. A. (1963). The structure of a semantic theory. Language, 39, 170-210.

Kim, A., \& Osterhout, L. (2005). The independence of combinatory semantic processing: Evidence from event-related potentials. Journal of Memory and Language, 52, 205-225.
Kutas, M., \& Federmeier, K. D. (2000). Electrophysiology reveals semantic memory use in language comprehension. Trends in Cognitive Sciences, 4, 463-470.

Kutas, M., \& Hillyard, S. A. (1980). Reading senseless sentences: Brain potentials reflect semantic incongruity. Science, 207, 203-205.

Kutas, M., \& Hillyard, S. A. (1984). Brain potentials during reading reflect word expectancy and semantic association. Nature, 307, 161-163.

Li, X., Hagoort, P., \& Yang, Y. (2008). Event-related potential evidence on the influence of accentuation in spoken discourse comprehension in Chinese. Journal of Cognitive Neuroscience, 20, 906-915.

Maris, E. (2004). Randomization tests for ERP topographies and whole spatiotemporal data matrices. Psychophysiology, 41, 142-151.

Maris, E., \& Oostenveld, R. (2007). Nonparametric statistical testing of EEG and MEG data. Journal of Neuroscience Methods, 164, 177-190.

McElree, B., Pylkkanen, L., Pickering, M. J., \& Traxler, M. J (2006). A time course analysis of enriched composition. Psychonomic Bulletin \& Review, 13, 53-59.

McElree, B., Traxler, M. J., Pickering, M. J., Seely, R. E., \& Jackendoff, R. (2001). Reading time evidence for enriched composition. Cognition, 78, 17-25.

Nieuwland, M. S., \& van Berkum, J. J. A. (2005). When peanuts fall in love: $\mathrm{N} 400$ evidence for the power of discourse. Journal of Cognitive Neuroscience, 18, 1098-1111.

Özyürek, A., Willems, R. M., Kita, S., \& Hagoort, P. (2007). On-line integration of semantic information from speech and gesture: Insights from event-related brain potentials. Journal of Cognitive Neuroscience, 19, 605-616.

Partee, B., ter Meulen, A., \& Wall, R. (1990). Mathematical methods in linguistics. Dordrecht: Kluwer.

Pustejovsky, J. (1995). The generative lexicon. Cambridge, MA: MIT Press.

Pylkkänen, L. (2008). Mismatching meanings in brain and behavior. Language and Linguistics Compass, 2/4, 712-738.

Pylkkänen, L., \& McElree, B. (2006). The syntax-semantics interface: On-line composition of sentence meaning. In M. J. Traxler \& M. A. Gernsbacher (Eds.), Handbook of psycholinguistics (2nd ed., pp. 537-577). New York: Elsevier.

Pylkkänen, L., \& McElree, B. (2007). An MEG study of silent meaning. Journal of Cognitive Neuroscience, 19, 1905-1921.

Rugg, M. D. (1983). Dissociation of semantic priming, word and non-word repetition effects by event-related potentials Quarterly Journal of Experimental Psychology A, 39, 123-148

Simos, P. G., Basile, L. F. H., \& Papanicolaou, A. C. (1997). Source localization of the N400 response in a sentence-reading paradigm using evoked magnetic fields and magnetic resonance imaging. Brain Research, 762, 29-39.

Traxler, M. J., McElree, B., Williams, R. S., \& Pickering, M. J. (2005). Context effects in coercion: Evidence from eye movements. Journal of Memory and Language, 53, 1-25.

Traxler, M. J., Pickering, M. J., \& McElree, B. (2002). Coercion in sentence processing: Evidence from eye-movements and self-paced reading. Journal of Memory and Language, 47, 530-547.

van Berkum, J., Hagoort, P., \& Brown, C. M. (1999). Semantic integration in sentences and discourse: Evidence from the N400. Journal of Cognitive Neuroscience, 11, 657-671. 
van Berkum, J., Zwitserlood, P., Hagoort, P., \& Brown, C. M. (2003). When and how do listeners relate a sentence to the wider discourse? Evidence from the N400 effect. Cognitive Brain Research, 17, 701-718.

van Berkum, J. J. A., Brown, C., \& Hagoort, P. (1999). Early referential context effects in sentence processing: Evidence from event-related brain potentials. Journal of Memory and Language, 41, 147-182.

van Berkum, J. J. A., Brown, C., Hagoort, P., \& Zwitserlood, P. (2003). Event-related brain potentials reflect discourse-referential ambiguity in spoken language comprehension. Psychophysiology, 40, 235-248. van Lambalgen, M., \& Hamm, F. (2004). The proper treatment of events. Oxford, UK: Blackwell.

van Petten, C., \& Kutas, M. (1990). Interactions between sentence context and word frequency in event-related brain potentials. Memory \& Cognition, 18, 380-393.

van Petten, C., \& Kutas, M. (1991). Influences of semantic and syntactic context on open- and closed-class words. Memory \& Cognition, 19, 95-112.

Vosse, T., \& Kempen, G. (2000). Syntactic structure assembly in human parsing: A computational model based on competitive inhibition and a lexicalist grammar. Cognition, 75, 105-143. 